\title{
Comparative analysis of lifestyle factors, screening test use, and clinicopathologic features in association with survival among Asian Americans with colorectal cancer
}

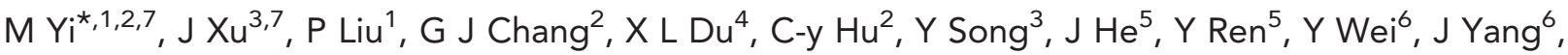
$\mathrm{K} \mathrm{K} \mathrm{Hunt}{ }^{2}$ and $\mathrm{X} \mathrm{Li}{ }^{1}$

${ }^{1}$ Department of Translational Medicine, The First Affiliated Hospital of Xian Jiaotong University, School of Medicine, Xian, China; ${ }^{2}$ Department of Surgical Oncology, The University of Texas MD Anderson Cancer Center, Houston, TX, USA; ${ }^{3}$ Department of Gastroenterology, Xi'an Central Hospital, Xi'an Jiaotong University, Xian, China; ${ }^{4}$ Division of Epidemiology and Disease Control, School of Public Health, The University of Texas Health Science Center at Houston, Houston, TX, USA; ${ }^{5}$ Department of Surgical Oncology, The First Affiliated Hospital of Xian Jiaotong University, School of Medicine, Xian, China and ${ }^{6}$ Department of Medical Oncology, The First Affiliated Hospital of Xian Jiaotong University, School of Medicine, Xian, China
\end{abstract}

Background: Colorectal cancer (CRC) diagnoses and disease-specific survival (DSS) vary between ethnic groups in the United States. However, few studies have assessed differences among Asian subgroups.

Methods: The Surveillance, Epidemiology, and End Results (SEER) database was used to identify patients with invasive CRC between 1988 and 2008. Differences in clinicopathologic features, and DSS rates were compared among Asian subgroups. The California Health Interview Survey was used to examine risk factors and screening patterns for CRC.

Results: The study included 359374 patients with 8.4\% Asian. Patients in all Asian subgroups were younger (median: 68 years) at diagnosis than non-Hispanic white (NHW) patients (median: 72 years). Most Asian subgroups, except Hawaiians, had better DSS than NHW patients although Asian subgroups had more advanced disease than NHW. Indian/Pakistani patients had a higher 5 -year DSS than other Asian subgroups. Obesity proportions were lower in Asian subgroups $(<50.2 \%)$ than in NHW (59.8\%). Vietnamese men and Korean women had the lowest proportions of CRC screening. Advance tumour stages were highly associated with worse DSS in each ethnicity group. High tumour grades were associated with worse DSS in NHW, Filipino, and Chinese. Older age at diagnosis was associated with worse DSS in most ethnicity groups except Hawaiian and Vietnamese.

Conclusion: Disparities exist between Asians and NHW with CRC, and among various Asian subgroups. Differences in cancer clinicopathologic features, patients' behavioural habits, lifestyle, and screening patterns may explain some differences in CRC survival observed among ethnic groups.

Asian Americans constitute the fastest growing ethnic group in the United States (American community survey, 2007). Colorectal cancer (CRC) is the third most common malignancy and the third most frequent cause of cancer death in the United States for overall population, although both incidence and mortality rates have declined in recent years (Cress et al, 2006).

\footnotetext{
*Correspondence: Dr M Yi; E-mail: myi@mdanderson.org

${ }^{7}$ These authors contributed equally to this work
}

Received 22 November 2012; revised 6 February 2013; accepted 12 February 2013; published online 7 March 2013 
Some studies have discussed the incidence of and mortality rates differences between Asian Americans and other ethnic groups, but few examined disparities among Asian subgroup populations (Chien et al, 2005; White et al, 2010). The purpose of this analysis was to determine whether there are significant differences in presentation, clinicopathologic features, treatment, and survival rates between non-Hispanic white (NHW) and Asian; and between Asian subgroups in patients with CRC. In addition, lifestyle factors and screening prevalence were analysed to determine whether any disparities exist between the Asian subgroups and other ethnic groups residing in the United States and to explore the potential associations among these difference.

\section{MATERIALS AND METHODS}

Patient selection and data collection. The Surveillance, Epidemiology, and End Results (SEER) database of the National Cancer Institute was used to identify patients who were diagnosed with invasive primary CRC between 1988 and 2008. Data were obtained from all 17 US cancer registries participating in the SEER programme using SEER $^{\star}$ Stat version 7.0.5 under a data user agreement (http://seer.cancer.gov/seerstat, access date: 23 January 2012). We included patients with invasive CRC with race coded as 'White' and 'Asian or Pacific Islander,' and 'Non-SpanishHispanic-Latino'.

Ethnicity was categorised into two broad groups: NHW and Asians (including Pacific Islanders). Asian ethnic groups were further categorised into the following eight subgroups: Filipino, Chinese, Japanese, Indian/Pakistani, Korean, Vietnamese, Hawaiian/Pacific Islander, and others.

Information on the prevalence of selected lifestyle factors for cancer and the use of CRC screening tests (colonoscopy and faecal occult blood testing) were derived from the 2009 California Health Interview Survey (CHIS) data using the askCHIS tool (http://ask.chis.ucla.edu/main/default.asp, access date: 17 February 2012). The CHIS is a state-wide telephone survey that was modelled after the National Health Interview Survey and was designed to provide population-based estimates for United States civilian, noninstitutionalised Californians. The information was only available for Chinese, Japanese, Korean, Filipino, and Vietnamese subgroups.

The $\chi^{2}$ test was used to assess differences in patient characteristics, patient management, and outcomes among the two broad ethnic groups and among the eight Asian subgroups. The primary end point of this study is disease-specific survival (DSS), which was calculated as the number of years between the date of diagnosis and the date of CRC-related death, date last known to be alive, or 31 December 2008. Patients not experiencing this end point were censored at last follow-up. Multivariate Cox proportional hazards models were used to determine the influence of patient, tumour, and treatment factors (age at diagnosis, gender, year of diagnosis, median household income, ethnicity, tumour stage, tumour grade, tumour primary site, and primary surgery) of known or potential prognostic value on DSS. Stata SE version 10.0 statistical software (StataCorp LP, College Station, TX, USA) was used for statistical analyses. All tests were two-tailed, and statistical significance was set at $P<0.05$.

\section{RESULTS}

Patient and tumour characteristics. We included 359374 patients with CRC: 329250 (91.6\%) NHWs and 30124 (8.4\%) Asians. Table 1 presents the comparisons among two broad ethnic groups (NHW and Asian). Asian patients were significantly
Table 1. Comparison of patient, tumour, and treatment characteristics among non-Hispanic white and Asian population in patients with colorectal cancer $(n=430625)$

\begin{tabular}{|l|c|c|}
\hline Characteristic & $\begin{array}{c}\text { Non-Hispanic white } \\
(n=329250)\end{array}$ & Asian $(n=30124)$ \\
\hline
\end{tabular}

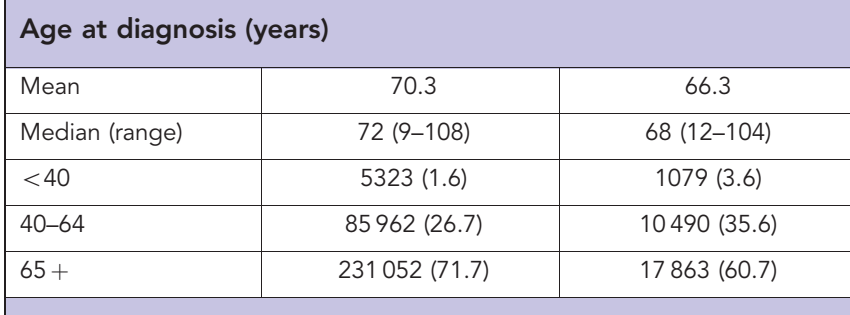

Sex

\begin{tabular}{|l|c|c|}
\hline Female & $162360(49.3)$ & $14235(47.2)$ \\
\hline Male & $166890(50.7)$ & $15889(52.8)$ \\
\hline
\end{tabular}

Marital status at diagnosis

\begin{tabular}{|l|c|c|}
\hline Married & $185941(58.4)$ & $19816(67.4)$ \\
\hline Other & $132494(41.6)$ & $9625(32.6)$ \\
\hline Unknown & 10815 & 683 \\
\hline Stage & $81480(24.7)$ & $6947(23.1)$ \\
\hline I & $99391(30.2)$ & $8234(27.3)$ \\
\hline II & $84983(25.8)$ & $9173(30.5)$ \\
\hline III & $63396(19.3)$ & $5770(19.1)$ \\
\hline IV & &
\end{tabular}

Surgery on primary

\begin{tabular}{|l|c|c|}
\hline Not performed & $23310(7.1)$ & $2038(6.8)$ \\
\hline Performed & $305678(92.9)$ & $28078(93.2)$ \\
\hline Unknown & 262 & 8 \\
\hline
\end{tabular}

Primary site

\begin{tabular}{|c|c|c|}
\hline Proximal colon & $164936(50.1)$ & $11855(39.4)$ \\
\hline Sigmoid colon & $70175(21.3)$ & $8375(27.8)$ \\
\hline Rectum & $83997(25.5)$ & $9185(30.5)$ \\
\hline Others & $10142(3.1)$ & 709 (2.3) \\
\hline \multicolumn{3}{|l|}{ Tumour grade } \\
\hline I & $29169(9.8)$ & $2126(7.7)$ \\
\hline II & $203161(68.2)$ & $20264(73.1)$ \\
\hline III & $62354(20.9)$ & $5168(18.6)$ \\
\hline Undifferentiated & $3387(1.1)$ & $172(0.6)$ \\
\hline Unknown & 31179 & 2394 \\
\hline \multicolumn{3}{|c|}{ Median household income (in $10 \$$ ) } \\
\hline Mean & 4784.4 & 5126.6 \\
\hline Median (range) & $4644(1581-7989)$ & 4982 (933-7989) \\
\hline
\end{tabular}

younger at diagnosis (median: 68 years) than NHW patients (median: 72 years). A greater percentage of Asian patients were diagnosed with stage III disease (30.5\%) than NHW (25.8\%).

Table 2 displays results from the comparisons among eight subgroups in the Asian cohort: Filipino (19.1\%), Japanese (26.9\%), Chinese (23.9\%), Hawaiian/Pacific Islander (6.9\%), Korean (7.5\%), Indian/Pakistani (3.0\%), Vietnamese (5.6\%), and others (7.1\%). Indian/Pakistani patients were the youngest at diagnosis (median age: 60.5 years) and Japanese patients were the oldest 
Table 2. Comparison of patient, tumour, and treatment characteristics among subgroups in the Asian cohort in patients with colorectal cancer $(n=30124)$

\begin{tabular}{|c|c|c|c|c|c|c|c|c|}
\hline Characteristic & $\begin{array}{c}\text { Filipino } \\
(n=5764)\end{array}$ & $\begin{array}{l}\text { Japanese } \\
(n=8105)\end{array}$ & $\begin{array}{c}\text { Chinese } \\
(n=7213)\end{array}$ & $\begin{array}{l}\text { Hawaiian } \\
(n=2070)\end{array}$ & $\begin{array}{c}\text { Korean } \\
(n=2257)\end{array}$ & $\begin{array}{c}\text { Indian/ } \\
\text { Pakistani } \\
(n=900)\end{array}$ & $\begin{array}{c}\text { Vietnamese } \\
(n=1690)\end{array}$ & $\begin{array}{c}\text { Other } \\
(n=2125)\end{array}$ \\
\hline
\end{tabular}

Age at diagnosis (years)

\begin{tabular}{|l|c|c|c|c|c|c|c|c|}
\hline Mean & 65.3 & 69.8 & 68.3 & 61.6 & 64.3 & 59.6 & 61.5 & 62.4 \\
\hline Median (range) & $66(12-100)$ & $71(23-100)$ & $70(17-102)$ & $62(12-96)$ & $65(17-97)$ & $60.5(18-96)$ & $62(19-98)$ & $63(14-97)$ \\
\hline$<40$ & $212(3.8)$ & $115(1.5)$ & $188(2.7)$ & $137(6.8)$ & $85(3.9)$ & $79(9.0)$ & $124(7.4)$ & $139(6.7)$ \\
\hline $40-64$ & $222(39.5)$ & $2076(26.3)$ & $2153(30.4)$ & $966(48.3)$ & $909(41.4)$ & $439(50.1)$ & $787(47.3)$ & $938(45.0)$ \\
\hline $65+$ & $3193(56.8)$ & $5710(72.3)$ & $4739(66.9)$ & $899(44.9)$ & $1201(54.7)$ & $359(40.9)$ & $754(45.3)$ & $1008(48.4)$ \\
\hline
\end{tabular}

Sex

\begin{tabular}{|l|c|c|c|c|c|c|c|c|}
\hline Female & $2599(45.1)$ & $3886(47.9)$ & $3487(48.3)$ & $914(44.1)$ & $1089(48.2)$ & $387(43.0)$ & $818(48.4)$ & $1055(49.6)$ \\
\hline Male & $3165(54.9)$ & $4219(52.1)$ & $3726(51.7)$ & $1156(55.9)$ & $1167(51.8)$ & $513(57.0)$ & $872(51.6)$ & $1070(50.3)$ \\
\hline
\end{tabular}

Marital status at diagnosis

\begin{tabular}{|l|c|c|c|c|c|c|c|c|}
\hline Married & $3921(69.5)$ & $5003(62.8)$ & $4972(70.5)$ & $1176(58.4)$ & $1573(71.3)$ & $667(76.3)$ & $1089(66.4)$ & $1415(69.0)$ \\
\hline Other & $1723(30.5)$ & $2958(37.2)$ & $2079(29.5)$ & $838(41.6)$ & $634(28.7)$ & $207(23.7)$ & $550(33.6)$ & $636(31.0)$ \\
\hline Unknown & 120 & 144 & 162 & 56 & 50 & 26 & 51 \\
\hline
\end{tabular}

\section{Stage}

\begin{tabular}{|c|c|c|c|c|c|c|c|c|}
\hline 1 & $1247(21.6)$ & $2013(24.8)$ & $1671(23.2)$ & $428(20.7)$ & $495(21.9)$ & $193(21.4)$ & $351(20.8)$ & $549(25.8)$ \\
\hline$\|$ & $1471(25.5)$ & $2268(28.0)$ & 2088 (28.9) & $514(26.1)$ & $612(27.1)$ & $246(27.3)$ & $475(28.1)$ & $533(25.1)$ \\
\hline III & 1809 (31.4) & $2459(30.3)$ & $2122(29.4)$ & $613(29.6)$ & $743(32.9)$ & $279(31.0)$ & $503(29.8)$ & 645 (30.4) \\
\hline IV & $1237(21.5)$ & $1365(16.8)$ & $1332(18.5)$ & $488(23.6)$ & 407 (18.1) & $182(20.2)$ & $361(21.3)$ & $398(18.7)$ \\
\hline \multicolumn{9}{|c|}{ Surgery on primary } \\
\hline Not performed & $492(8.5)$ & $436(5.4)$ & $426(5.9)$ & $183(8.8)$ & $147(6.5)$ & $76(8.4)$ & $109(6.4)$ & $169(8.0)$ \\
\hline Performed & 5269 (91.5) & 7669 (94.6) & $6785(94.1)$ & $1886(91.2)$ & 2109 (93.5) & $824(91.6)$ & $1581(93.6)$ & $1955(92.0)$ \\
\hline Unknown & 3 & 0 & 0 & 1 & 1 & 0 & 0 & 1 \\
\hline \multicolumn{9}{|l|}{ Primary site } \\
\hline Proximal colon & 1907 (33.1) & $3499(43.2)$ & $3033(42.1)$ & $802(38.7)$ & 807 (35.7) & $331(36.8)$ & $655(38.8)$ & $821(38.6)$ \\
\hline Sigmoid colon & $1726(29.9)$ & $2190(27.0)$ & $2028(28.1)$ & $524(25.3)$ & $606(26.9)$ & $239(26.6)$ & $457(27.0)$ & $605(28.5)$ \\
\hline Rectum & $1987(34.5)$ & $2254(27.8)$ & $1999(27.7)$ & $672(32.5)$ & 795 (35.2) & 299 (33.2) & 539 (31.9) & $640(30.1)$ \\
\hline Others & $144(2.5)$ & $162(2.0)$ & $153(2.1)$ & $72(3.5)$ & $49(2.2)$ & $31(3.4)$ & $39(2.3)$ & $59(2.8)$ \\
\hline \multicolumn{9}{|l|}{ Tumour grade } \\
\hline 1 & $425(8.1)$ & $567(7.5)$ & $457(6.9)$ & $147(7.8)$ & $175(8.3)$ & $81(9.8)$ & $102(6.5)$ & $172(8.9)$ \\
\hline II & 3807 (72.1) & $5766(76.6)$ & 4825 (72.8) & $1413(75.1)$ & $1463(69.9)$ & $565(68.3)$ & $1088(69.7)$ & $1339(69.5)$ \\
\hline III & $1.011(19.1)$ & $1167(15.5)$ & 1295 (19.5) & $314(16.7)$ & $444(21.2)$ & $172(20.8)$ & $361(23.2)$ & $404(21.0)$ \\
\hline Undifferentiated & $37(0.7)$ & $30(0.4)$ & $53(0.8)$ & $8(0.4)$ & $12(0.6)$ & $9(1.1)$ & $10(0.6)$ & $13(0.7)$ \\
\hline Unknown & 484 & 575 & 583 & 188 & 163 & 73 & 131 & 197 \\
\hline \multicolumn{9}{|c|}{ Median household income (in 10 \$) } \\
\hline Mean & 5133.8 & 4974.8 & 5299.9 & 4936.9 & 4896.2 & 5456.6 & 5443.5 & 5155.5 \\
\hline Median (range) & $\begin{array}{c}4982 \\
(2426-7734)\end{array}$ & $\begin{array}{c}4982 \\
(1956-7989)\end{array}$ & $\begin{array}{c}5522 \\
(2274-7989)\end{array}$ & $\begin{array}{c}4982 \\
(933-7734)\end{array}$ & $\begin{array}{c}4289 \\
(2986-7989)\end{array}$ & $\begin{array}{c}5595 \\
(2315-7989)\end{array}$ & $\begin{array}{c}5316 \\
(2713-7734)\end{array}$ & $\begin{array}{c}5191 \\
(1906-7989)\end{array}$ \\
\hline
\end{tabular}

(median age: 71 years). The proportion of patients who had proximal colon cancer was much higher in the Japanese group $(43.2 \%)$ than in other subgroups.

Risk factors and screening behaviours. Overweight and obesity proportions in Asian subgroups were much lower than in NHW (Table 3). The CRC screening proportions in all Asian subgroups were lower than in NHW. Smoking (current or former) was most prevalent in Korean men (56.6\%). Alcohol use was much higher in NHW men (39.9\%) and Korean men $(36.5 \%)$. Vietnamese men had lower proportions of screening tests $(52.2 \%)$ than other Asian groups. Korean women had lower proportions of screening tests $(56.7 \%)$ than other Asian groups. 
Table 3. Prevalence of risk factors, screening behaviours, and health care access among Asian American ethnic groups versus NHW in California ${ }^{a}$, stratified by gender

\section{Chinese}

Japanese

Korean

Filipino

Vietnamese

NHW

\section{Male}

\section{Smoking status}

\begin{tabular}{|l|l|l|l|l|l|}
\hline Current & $8.2 \%$ & $10.1 \%$ & $21.5 \%$ & $18.6 \%$ & $30.5 \%$ \\
\hline Former & $15.6 \%$ & $27.5 \%$ & $35.1 \%$ & $22.0 \%$ & $32.9 \%$ \\
\hline Never $^{\wedge}$ & $76.3 \%$ & $62.3 \%$ & $43.4 \%$ & $59.4 \%$ & $50.1 \%$ \\
\hline
\end{tabular}

\section{Alcohol}

\begin{tabular}{|c|c|c|c|c|c|c|}
\hline Drinker & $19.7 \%$ & $12.3 \%$ & $35.5 \%$ & $34.8 \%$ & $22.5 \%$ & $39.9 \%$ \\
\hline None in 1 year & $80.3 \%$ & $87.7 \%$ & 64.50 & $65.2 \%$ & $77.5 \%$ & $60.1 \%$ \\
\hline \multicolumn{7}{|l|}{ BMI } \\
\hline$<25$ & $70.9 \%$ & $52.4 \%$ & $58.9 \%$ & $49.9 \%$ & $75.1 \%$ & $40.2 \%$ \\
\hline$\geqslant 25$ & $29.1 \%$ & $47.6 \%$ & $41.1 \%$ & $50.1 \%$ & $24.9 \%$ & $59.8 \%$ \\
\hline
\end{tabular}

\section{Level of physical activity ${ }^{a}$}

\begin{tabular}{|l|l|l|l|l|l|l|}
\hline No & $18.1 \%$ & $12.5 \%$ & $15.6 \%$ & $14.0 \%$ & $23.7 \%$ & $11.3 \%$ \\
\hline Some & $52.7 \%$ & $48.5 \%$ & $53.4 \%$ & $48.1 \%$ & $54.5 \%$ & $46.8 \%$ \\
\hline Moderate & $15.1 \%$ & $17.7 \%$ & $12.3 \%$ & $13.2 \%$ & $11.2 \%$ & $19.1 \%$ \\
\hline Vigorous & $14.2 \%$ & $21.3 \%$ & $18.6 \%$ & $24.7 \%$ & $18.0 \%$ & $22.9 \%$ \\
\hline
\end{tabular}

\section{Ever had sigmoidoscopy, colonoscopy, or FOBT ${ }^{\mathrm{a}}$}

\begin{tabular}{|l|l|l|l|l|l|l|}
\hline Never had one of them & $18.7 \%$ & $21.8 \%$ & $27.6 \%$ & $31.3 \%$ & $47.8 \%$ & $16.5 \%$ \\
\hline Have had one of them & $81.3 \%$ & $78.2 \%$ & $72.4 \%$ & $68.7 \%$ & $52.2 \%$ & $83.5 \%$ \\
\hline
\end{tabular}

\section{Type of most recent CRC screening}

\begin{tabular}{|l|c|c|c|c|r|r|}
\hline Colonoscopy & $66.2 \%$ & $55.3 \%$ & $66.4 \%$ & $45.5 \%$ & $49.7 \%$ & $56.4 \%$ \\
\hline Sigmoidoscopy & $11.1 \%$ & $4.9 \%$ & - & $11.5 \%$ & $4.7 \%$ & $10.8 \%$ \\
\hline FOBT & $22.7 \%$ & $39.8 \%$ & $31.3 \%$ & $43.0 \%$ & $45.6 \%$ & $32.8 \%$ \\
\hline
\end{tabular}

\section{CRC screening compliance at time of recommendation}

\begin{tabular}{|c|c|c|c|c|c|c|}
\hline Not compliant & $25.7 \%$ & $34.6 \%$ & $31.3 \%$ & $43.5 \%$ & $50.0 \%$ & $26.6 \%$ \\
\hline Compliant & $74.3 \%$ & $65.4 \%$ & $68.7 \%$ & $56.5 \%$ & $50.0 \%$ & $73.4 \%$ \\
\hline \multicolumn{7}{|c|}{ Health insurance } \\
\hline Currently insured & $90.3 \%$ & $93.5 \%$ & $70.1 \%$ & $82.9 \%$ & $90.2 \%$ & $91.4 \%$ \\
\hline Not & $9.7 \%$ & $6.5 \%$ & $29.9 \%$ & $17.1 \%$ & $9.8 \%$ & $8.6 \%$ \\
\hline \multicolumn{7}{|c|}{ Ever diagnosed with diabetes } \\
\hline Yes & $7.1 \%$ & $12.5 \%$ & $7.3 \%$ & $16.1 \%$ & $4.1 \%$ & $7.1 \%$ \\
\hline No & $92.9 \%$ & $87.5 \%$ & $92.7 \%$ & $83.9 \%$ & $95.9 \%$ & $92.9 \%$ \\
\hline \multicolumn{7}{|l|}{ Female } \\
\hline \multicolumn{7}{|l|}{ Smoking status } \\
\hline Current & $2.9 \%$ & $6.9 \%$ & $9.5 \%$ & $4.6 \%$ & $2.0 \%$ & $12.4 \%$ \\
\hline Former & $5.3 \%$ & $18.3 \%$ & $8.6 \%$ & $9.2 \%$ & $1.0 \%$ & $27.4 \%$ \\
\hline Never^ & $91.9 \%$ & $74.8 \%$ & $81.9 \%$ & $86.3 \%$ & $97.0 \%$ & $60.2 \%$ \\
\hline \multicolumn{7}{|l|}{ Alcohol } \\
\hline Drinker & $5.9 \%$ & $9.0 \%$ & $17.6 \%$ & $16.9 \%$ & $14.8 \%$ & $25.7 \%$ \\
\hline None in 1 year & $94.1 \%$ & $91.0 \%$ & $82.4 \%$ & $83.1 \%$ & $85.2 \%$ & $74.3 \%$ \\
\hline
\end{tabular}




\begin{tabular}{|c|c|c|c|c|c|c|}
\hline & Chinese & Japanese & Korean & Filipino & Vietnamese & NHW \\
\hline \multicolumn{7}{|c|}{ Level of physical activity ${ }^{a}$} \\
\hline No & $23.6 \%$ & $22.2 \%$ & $25.6 \%$ & $13.8 \%$ & $12.6 \%$ & $12.2 \%$ \\
\hline Some & $53.5 \%$ & $43.5 \%$ & $49.0 \%$ & $53.1 \%$ & $53.3 \%$ & $56.5 \%$ \\
\hline Moderate & $18.5 \%$ & $19.8 \%$ & $18.7 \%$ & $17.2 \%$ & $20.1 \%$ & $18.6 \%$ \\
\hline Vigorous & $4.4 \%$ & $14.5 \%$ & $6.7 \%$ & $15.9 \%$ & $14.0 \%$ & $12.7 \%$ \\
\hline \multicolumn{7}{|l|}{ BMI } \\
\hline$<25$ & $84.3 \%$ & $71.2 \%$ & $83.9 \%$ & $70.2 \%$ & $84.7 \%$ & $58.4 \%$ \\
\hline$\geqslant 25$ & $15.7 \%$ & $28.8 \%$ & $16.1 \%$ & $29.8 \%$ & $15.3 \%$ & $41.6 \%$ \\
\hline \multicolumn{7}{|c|}{ Ever had sigmoidoscopy, colonoscopy, or $\mathrm{FOBT}^{\mathrm{a}}$} \\
\hline Never had one of them & $39.6 \%$ & $21.4 \%$ & $43.3 \%$ & $19.8 \%$ & $39.4 \%$ & $18.5 \%$ \\
\hline Have had one of them & $60.4 \%$ & $78.6 \%$ & $56.7 \%$ & $80.2 \%$ & $60.6 \%$ & $81.5 \%$ \\
\hline \multicolumn{7}{|c|}{ Type of most recent CRC screening } \\
\hline Colonoscopy & $44.9 \%$ & $47.6 \%$ & $64.1 \%$ & $39.6 \%$ & $44.5 \%$ & $56.5 \%$ \\
\hline Sigmoidoscopy & $15.1 \%$ & $8.1 \%$ & $6.9 \%$ & $15.2 \%$ & $25.2 \%$ & $8.3 \%$ \\
\hline FOBT & $40.0 \%$ & $44.3 \%$ & $28.9 \%$ & $45.2 \%$ & $30.3 \%$ & $35.2 \%$ \\
\hline \multicolumn{7}{|c|}{ CRC screening compliance at time of recommendation } \\
\hline Not compliant & $36.7 \%$ & $24.3 \%$ & $44.6 \%$ & $35.6 \%$ & $25.7 \%$ & $29.4 \%$ \\
\hline Compliant & $63.3 \%$ & $75.7 \%$ & $55.4 \%$ & $64.4 \%$ & $74.3 \%$ & $70.6 \%$ \\
\hline \multicolumn{7}{|l|}{ Health insurance } \\
\hline Currently insured & $90.9 \%$ & $99.0 \%$ & $77.2 \%$ & $97.2 \%$ & $84.2 \%$ & $93.2 \%$ \\
\hline Not & $9.1 \%$ & $1.0 \%$ & $22.8 \%$ & $2.8 \%$ & $15.8 \%$ & $6.8 \%$ \\
\hline \multicolumn{7}{|c|}{ Ever diagnosed with diabetes } \\
\hline Yes & $6.8 \%$ & $8.2 \%$ & $6.4 \%$ & $10.2 \%$ & $3.0 \%$ & $5.5 \%$ \\
\hline No & $93.2 \%$ & $91.8 \%$ & $93.6 \%$ & $89.8 \%$ & $97.0 \%$ & $94.5 \%$ \\
\hline
\end{tabular}

Survival. Overall, Asian had better DSS than did NHW (Hazard ratio (HR) $0.95, P=0.001$ ). Most Asian subgroups (Indian/ Pakistani, HR 0.68, $P<0.0001$, Chinese, HR 0.94, $P=0.025$, and Japanese HR 0.93, $P=0.006$,), except Hawaiian (HR 1.2, $P<0.0001$ ), had better DSS than did NHW patients. Table 4 shows the association between disease stage, tumour grade, sex, primary site of tumour, status of surgery, year of diagnosis, age at diagnosis, and median household income with DSS in each ethnicity group. Patients with distal sigmoid colon cancer had better DSS in Filipino (HR 0.8, $P=0.03$ ). Advance tumour stages were highly associated with worse DSS in each ethnicity group. High tumour grades were associated with worse DSS in NHW, Filipino and Chinese. Older age at diagnosis was associated with worse DSS in most ethnicity groups except Hawaiian and Vietnamese. Higher income was associated with better DSS only in NHW and Hawaiian.

\section{DISCUSSION}

This study is one of the most comprehensive population-based analyses of CRC by ethnicity reported in the literature, and looked at not only clinicopathologic factors (with 359374 cases included) and also the lifestyle and screening data. Many of our findings confirm previous less comprehensive studies including: Asian groups' lower use of screening (Fenton et al, 2009); Asian patients presented with more advanced disease than NHW (Ayanian, 2010; Robbins et al, 2012); a younger average age at diagnosis in Asian patients (Koo et al, 2008; Norwood et al, 2009; Sammour et al, 2010).

Racial/ethnic disparities in CRC survival has been extensively documented in the scientific literature (Chien et al, 2005; Fenton et al, 2009; Robbins et al, 2012). We found that most Asian patients had better DSS than did NHW. Differences in tumour site distribution and genetics may explain the high survival rates observed among Asians. That is, relative to other groups, Asians have higher rates of distal colon cancer, which is associated with a decreased risk of mortality. This further confirms the findings of previous studies (Le et al, 2009). We also found that Indian/ Pakistani patients had better DSS than did other groups even after adjusted by age, tumour stage, grade, and tumour site. According to US census data, Asian Indians are the wealthiest major ethnic group in the country (Goggins and Wong, 2009). Thus, it is reasonable to hypothesise that the relatively higher CRC survival for Indian/Pakistani patients results from better treatment. 


\begin{tabular}{|c|c|c|c|c|c|c|c|c|c|c|c|c|c|c|c|c|c|c|c|c|c|c|c|}
\hline \multirow[t]{2}{*}{ 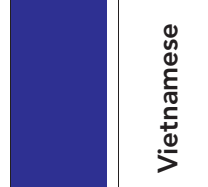 } & $\begin{array}{l}\frac{0}{\pi} \\
\frac{3}{1} \\
a^{2}\end{array}$ & & & $\begin{array}{l}\text { o̊ } \\
0 \\
0\end{array}$ & $\bar{\delta}$. & $\begin{array}{l}\bar{\delta} \\
\dot{0} \\
\text { Q }\end{array}$ & & & 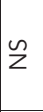 & $\tilde{z}$ & $\tilde{z}$ & $\sum$ & & & $z \quad \frac{u}{2}$ & 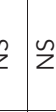 & & & $\Sigma$ & $\sum$ & 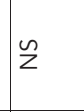 & $\tilde{z}$ & \\
\hline & $\stackrel{\propto}{I}$ & & & $\stackrel{\leftrightarrow}{\mathrm{\sim}}$ & טִ & o. & & & & & & & & & & & & & & & & & \\
\hline 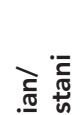 & $\frac{\frac{0}{3}}{\frac{3}{\pi}}$ & & & ○̊ & $\begin{array}{l}\bar{\delta} \\
0 \\
0 \\
v\end{array}$ & $\begin{array}{l}\bar{\delta} \\
\text { o. } \\
\text { v }\end{array}$ & & & 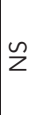 & $\tilde{z}$ & $\tilde{z}$ & ¿ & & & \{ & $\tilde{z}$ & & & $\tilde{z}$ & 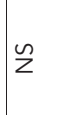 & $\tilde{z}$ & סू. & \\
\hline$\leq$ & $\frac{\alpha}{I}$ & & & $\stackrel{\sim}{\sim}$ & 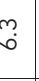 & :ُ & & & & & & & & & & & & & & & & $\stackrel{m}{\stackrel{\rho}{\circ}}$ & \\
\hline ฐ & $\frac{\frac{0}{2}}{\frac{\pi}{\pi}}$ & & & o̊ & $\begin{array}{l}\bar{b} \\
\text { o. } \\
\text { v }\end{array}$ & $\begin{array}{c}\bar{\delta} \\
0 \\
0 \\
v\end{array}$ & & & 范 & $\tilde{z}$ & $\tilde{z}$ & $\tilde{z}$ & & & 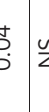 & $z \sum$ & & & $\begin{array}{l}\infty \\
0 \\
0 \\
0\end{array}$ & চ̄ & $\tilde{z}$ & $\begin{array}{l}\bar{\delta} \\
\dot{o} \\
\dot{0} \\
v\end{array}$ & \\
\hline$\underline{x}$ & 趿 & & & $\stackrel{\rho}{-}$ & 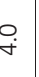 & $\begin{array}{l}n^{3} \\
\stackrel{0}{\circ}\end{array}$ & & & $\cong$ & & & & & & ? & & & & $\hat{o}$ & $\stackrel{\sim}{\circ}$ & & $\underset{\sim}{\sigma}$ & \\
\hline$\stackrel{\frac{c}{\mathbb{\pi}}}{:}$ & $\frac{\frac{0}{2}}{\frac{\pi}{\pi}}$ & & & $\bar{\delta}$ & $\begin{array}{l}\overline{\text { og }} \\
\text { i } \\
\text { v }\end{array}$ & $\begin{array}{c}\bar{\delta} \\
0 \\
0 \\
v\end{array}$ & & & סे & $\tilde{z}$ & $\tilde{z}$ & $\tilde{z}$ & & & $z \frac{4}{2}$ & $z \bumpeq$ & & & $\begin{array}{l}\bar{\delta} \\
0 \\
\dot{v} \\
v\end{array}$ & $\tilde{z}$ & ठ̃ & $\tilde{z}$ & \\
\hline$\underset{\substack{3 \\
10}}{3}$ & 吕 & & & $\bar{i}$ & $\begin{array}{c}\text { Ny } \\
\text { ñ }\end{array}$ & & & & $\stackrel{\cong}{=}$ & & & & & & & & & & $\hat{0}$ & & \begin{tabular}{l}
$\infty$ \\
\hdashline \\
$\alpha$ \\
0 \\
0
\end{tabular} & & \\
\hline$\stackrel{\Xi}{\mathscr{\Xi}}$ & $\frac{0}{\frac{0}{\pi}}$ & & & $\begin{array}{l}\bar{\delta} \\
\dot{0} \\
\dot{v}\end{array}$ & $\begin{array}{l}\bar{\delta} \\
\dot{\delta} \\
\dot{v} \\
v\end{array}$ & $\begin{array}{l}\bar{\delta} \\
\text { o. } \\
\text { v }\end{array}$ & & & $\tilde{z}$ & $\begin{array}{l}\tilde{O} \\
\text { ô } \\
0\end{array}$ & 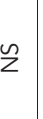 & n & & & $z \quad \frac{u}{2}$ & $z \sum$ & & & $\begin{array}{l}\bar{\delta} \\
\dot{0} \\
\bar{v}\end{array}$ & $\begin{array}{l}\bar{z} \\
\dot{o} \\
\dot{v}\end{array}$ & $\check{z}$ & $\begin{array}{l}\bar{\delta} \\
\dot{0} \\
\dot{0} \\
v\end{array}$ & \\
\hline $\bar{U}$ & 哇 & & & $\stackrel{\circ}{\mathrm{i}}$ & $\stackrel{\substack{* \\
\sim}}{+}$ & $\begin{array}{l}\infty \\
\infty \\
\stackrel{\infty}{\infty}\end{array}$ & & & & $\stackrel{\circ}{\circ}$ & & & & & & & & & 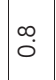 & $\stackrel{\circ}{\circ}$ & & $\stackrel{\sigma}{-}$ & \\
\hline$\stackrel{\Xi}{\Perp}$ & $\frac{\frac{0}{3}}{\frac{\pi}{0}}$ & & & $\begin{array}{l}\bar{\delta} \\
\dot{0} \\
\dot{v}\end{array}$ & $\begin{array}{l}\bar{\delta} \\
\dot{\delta} \\
\dot{v} \\
v\end{array}$ & $\begin{array}{c}\bar{\delta} \\
0 \\
0\end{array}$ & & & 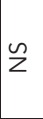 & $\tilde{z}$ & $\tilde{z}$ & $\begin{array}{l}\overline{0} \\
0 \\
0\end{array}$ & & & $z$ & 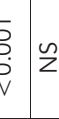 & & & $\begin{array}{l}\bar{\delta} \\
\bar{o} \\
\dot{v}\end{array}$ & $\begin{array}{l}\bar{\delta} \\
\dot{0} \\
\dot{v}\end{array}$ & $\tilde{z}$ & $\begin{array}{l}\bar{\delta} \\
\dot{D} \\
\dot{Q} \\
\text { v }\end{array}$ & \\
\hline$\stackrel{\frac{2}{10}}{7}$ & 呈 & & & $\stackrel{\text { ì }}{\mathrm{i}}$ & $\begin{array}{l}\infty \\
\stackrel{\infty}{+}\end{array}$ & ָ̃ & & & & & & 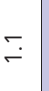 & & & ? & $\underline{y}$ & & & $\begin{array}{l}a \\
\hat{0}\end{array}$ & 色 & & 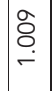 & \\
\hline 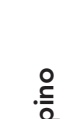 & $\frac{\frac{0}{3}}{\frac{\pi}{01}}$ & & & $\begin{array}{l}\bar{\delta} \\
\dot{0} \\
\dot{v} \\
v\end{array}$ & $\begin{array}{l}\bar{\delta} \\
\dot{0} \\
v\end{array}$ & $\overline{8}$ & & & $\tilde{z}$ & ô & $\Sigma$ & 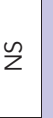 & & & 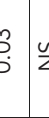 & $z=$ & & & $\begin{array}{l}m \\
0 \\
0 \\
0\end{array}$ & 0 & $\tilde{z}$ & o. & \\
\hline$\overline{\bar{i}}$ & $\frac{\mathscr{\alpha}}{I}$ & & & $\stackrel{\sim}{\sim}$ & $\bar{i}$ & $\begin{array}{c}m \\
\infty \\
\infty\end{array}$ & & & & $\stackrel{n}{\leftarrow}$ & & & & & b. & i. & & & $\begin{array}{l}\infty \\
0 \\
0\end{array}$ & $\stackrel{\circ}{\circ}$ & & 岂 & \\
\hline & $\frac{\frac{0}{2}}{\sum_{0}^{\pi}}$ & & & $\begin{array}{l}\bar{\delta} \\
\dot{d} \\
\dot{0}\end{array}$ & $\begin{array}{l}\bar{\delta} \\
\dot{0} \\
\dot{v}\end{array}$ & $\begin{array}{c}\overline{0} \\
0 \\
0 \\
0\end{array}$ & & & 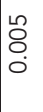 & $\begin{array}{l}\bar{\delta} \\
\dot{0} \\
\dot{v} \\
\end{array}$ & 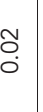 & 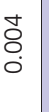 & & & $\begin{array}{l}v \\
D \\
D\end{array}$ & $\dot{D}^{5}$ & & & $\begin{array}{l}\bar{\delta} \\
\dot{0} \\
\dot{v}\end{array}$ & $\begin{array}{l}\bar{\partial} \\
\bar{\alpha} \\
\dot{v}\end{array}$ & ¿. & $\begin{array}{l}\bar{\delta} \\
\dot{0} \\
\dot{v}\end{array}$ & 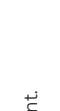 \\
\hline & $\frac{\alpha}{I}$ & & & $\bar{i}$ & 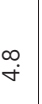 & $\stackrel{n}{\alpha}$ & & & $\stackrel{\sim}{\longrightarrow}$ & $\stackrel{\llcorner}{\longrightarrow}$ & $\stackrel{\circ}{\circ}$ & $\check{F}$ & & & 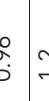 & $\underline{y}$ & & & $\begin{array}{c}\infty \\
0 \\
0\end{array}$ & $\because$ & $\begin{array}{l}\alpha \\
\alpha \\
\alpha \\
\sigma \\
\sigma\end{array}$ & $\underset{\sigma}{\sigma}$ & 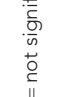 \\
\hline & $\frac{\frac{0}{2}}{\frac{\pi}{30}}$ & & & $\begin{array}{l}\bar{\delta} \\
\dot{0} \\
\dot{v}\end{array}$ & $\begin{array}{l}\bar{o} \\
\text { Q } \\
\text { v }\end{array}$ & ¿. & & & $\begin{array}{l}\bar{\delta} \\
\dot{0} \\
\dot{0} \\
\text { v }\end{array}$ & $\begin{array}{l}\bar{\delta} \\
\dot{0} \\
\dot{v} \\
v\end{array}$ & $\begin{array}{l}\bar{\delta} \\
\dot{0} \\
\mathrm{v}\end{array}$ & $\begin{array}{l}\bar{\delta} \\
\dot{0} \\
\dot{0} \\
v\end{array}$ & & & $\bar{D}$ & 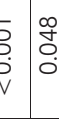 & & & $\begin{array}{l}\bar{\delta} \\
\dot{0} \\
v\end{array}$ & $\begin{array}{l}\bar{\delta} \\
0 \\
0 \\
v\end{array}$ & $\begin{array}{l}\bar{o} \\
\dot{0} \\
\mathrm{v}\end{array}$ & $\begin{array}{l}\bar{\delta} \\
0 \\
\dot{0}\end{array}$ & 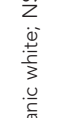 \\
\hline$\frac{T}{z}$ & $\underline{\alpha} \underline{\underline{I}}$ & & 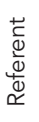 & $\bar{i}$ & $\hat{f}$ & $\stackrel{m}{s}$ & & 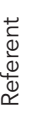 & 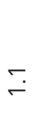 & $\stackrel{m}{\rightarrow}$ & $\stackrel{\sim}{\sim}$ & $\check{\sigma}$ & & 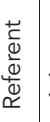 & $=$ & $\stackrel{n}{-}$ & & 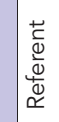 & $\underset{\infty}{\infty}$ & $\hat{o}$ & $\begin{array}{l}\infty \\
\alpha \\
\alpha \\
\alpha \\
\alpha \\
0\end{array}$ & $\underset{-}{-}$ & 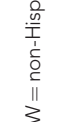 \\
\hline & $\begin{array}{l}\frac{0}{0} \\
\frac{0}{\frac{\pi}{0}} \\
\frac{0}{\pi}\end{array}$ & $\frac{1}{g}$ & - & $=$ & $\equiv$ & $\geq$ & & $\frac{\bar{\sigma}}{\bar{\alpha}}$ & $\begin{array}{l}= \\
\frac{0}{0} \\
\frac{0}{0} \\
\frac{0}{0} \\
\end{array}$ & 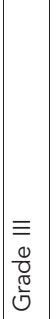 & $\begin{array}{l}\geq \\
\frac{0}{0} \\
\frac{0}{0} \\
\end{array}$ & 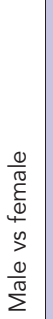 & 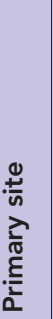 & 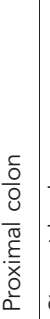 & 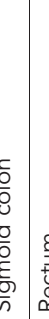 & 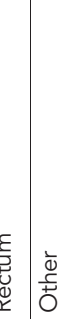 & 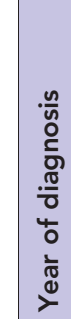 & 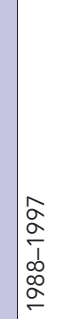 & 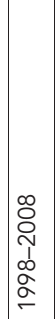 & 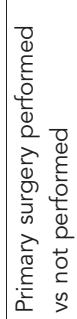 & 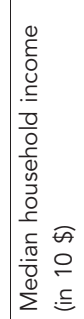 & 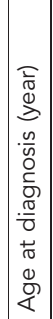 & 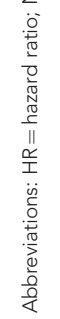 \\
\hline
\end{tabular}


Lifestyle differences may also explain some of differences in CRC survival. Obese individuals have higher morbidity and mortality for many cancers including CRC (Cress et al, 2006). Obese patients have a $50 \%$ increased risk of developing CRC and a 30\% higher risk of dying from CRC than non-obese patients (Calle et al, 2003). Moreover, obese patients treated for CRC have poorer OS than normal-weight patients (White et al, 2010). According to our study, overweight and obesity proportions in Asian subgroups were much lower than in NHW. This might be another contributing factor to better survival in Asian groups.

Regarding aetiologic factors, high alcohol intake has been consistently associated with increased risk and moderate or high physical activity with decreased risk of CRC, and these factors vary by race, sex, and socioeconomic status (Murphy et al, 2011). Smoking also appears to be a strong risk factor for CRC. In our study, alcohol use was most prevalent in NHW men and Korean men and smoking was most prevalent in Korean men. In contrast to the decreasing trends in CRC incidence reported among all major racial/ethnic groups, CRC rates are actually increasing among some Asian subgroups, including the Korean population (Giddings et al, 2012). Relatively high alcohol consumption and high smoking proportions in the Korean community might be related to the significant increase in CRC incidence.

Some limitations should be considered in the interpretation of this study. First, this study was limited by its use of national administrative databases. A lack of data on the individual level socioeconomic status and the administration of systemic therapies limited us from evaluating these factors as potential confounders. Second, the lifestyle and screening data were obtained only from California and included only five Asian subgroups, which may not represent the status of entire United States. However, most Asians in SEER are from California registries.

\section{CONCLUSION}

Our results, which are based on a large, national population-based sample, show that there are differences in presenting clinicopathologic features between CRC patients of different races/ethnicities of in the United States and that these differences may affect survival.

\section{REFERENCES}

American community survey 2007 (2007) A Comparison to Selected Housing and Financial Characteristics for the United States from the 2007 American Housing Survey Vol. 2011US Census Bureau: Washington.

Ayanian JZ (2010) Racial disparities in outcomes of colorectal cancer screening: biology or barriers to optimal care? J Natl Cancer Inst 102(8): 511-513.

Calle EE, Rodriguez C, Walker-Thurmond K, Thun MJ (2003) Overweight, obesity, and mortality from cancer in a prospectively studied cohort of U.S. adults. N Engl J Med 348(17): 1625-1638.

Chien C, Morimoto LM, Tom J, Li CI (2005) Differences in colorectal carcinoma stage and survival by race and ethnicity. Cancer 104(3): 629-639.

Cress RD, Morris C, Ellison GL, Goodman MT (2006) Secular changes in colorectal cancer incidence by subsite, stage at diagnosis, and race/ethnicity, 1992-2001. Cancer 107(5 Suppl): 1142-1152.

Fenton JJ, Tancredi DJ, Green P, Franks P, Baldwin LM (2009) Persistent racial and ethnic disparities in up-to-date colorectal cancer testing in medicare enrollees. J Am Geriatr Soc 57(3): 412-418.

Giddings BH, Kwong SL, Parikh-Patel A, Bates JH, Snipes KP (2012) Going against the tide: increasing incidence of colorectal cancer among Koreans, Filipinos, and South Asians in California, 1988-2007. Cancer Causes Control 23(5): 691-702.

Goggins WB, Wong G (2009) Cancer among Asian Indians/Pakistanis living in the United States: low incidence and generally above average survival. Cancer Causes Control 20(5): 635-643.

Koo JH, Kin S, Wong C, Jalaludin B, Kneebone A, Connor SJ, Leong RW (2008) Clinical and pathologic outcomes of colorectal cancer in a multiethnic population. Clin Gastroenterol Hepatol 6(9): 1016-1021.

Le H, Ziogas A, Taylor TH, Lipkin SM, Zell JA (2009) Survival of distinct Asian groups among colorectal cancer cases in California. Cancer 115(2): 259-270.

Murphy G, Devesa SS, Cross AJ, Inskip PD, McGlynn KA, Cook MB (2011) Sex disparities in colorectal cancer incidence by anatomic subsite, race and age. Int J Cancer 128(7): 1668-1675.

Norwood MG, Mann CD, Hemingway D, Miller AS (2009) Colorectal cancer: presentation and outcome in British South Asians. Colorectal Dis 11(7): 745-749.

Robbins AS, Siegel RL, Jemal A (2012) Racial disparities in stage-specific colorectal cancer mortality rates from 1985 to 2008. J Clin Oncol 30(4): 401-405.

Sammour T, Kahokehr A, Vather R, Connolly AB, Hill AG (2010) Ethnic disparity in colonic cancer outcomes in New Zealand-biology or an access issue? Colorectal Dis 12(7 Online): e50-e56.

White A, Vernon SW, Franzini L, Du XL (2010) Racial disparities in colorectal cancer survival: to what extent are racial disparities explained by differences in treatment, tumour characteristics, or hospital characteristics? Cancer 116(19): 4622-4631. 